\title{
A Psychological Adaptive Model For Video Analysis
}

\author{
N. Ghosh and B. Bhanu \\ Center for Research in Intelligent Systems (CRIS), University of California, Riverside, CA 92521, USA \\ \{nirmalya,bhanu\}@ee.ucr.edu
}

\begin{abstract}
Extracting key-frames is the first step for efficient content-based indexing, browsing and retrieval of the video data in commercial movies. Most of the existing research deals with "how to extract representative frames?" However the unaddressed question is "how many key-frames are required to represent a video shot properly?" Generally, the user defines this number a priori or some heuristic methods are used. In this paper, we propose a psychological model, which computes this number adaptively and online, from variation of visual features in a video-shot. We incorporate it with an iterative key-frame selection method to automatically select the key-frames. We compare the results of this method with two other well-known approaches, based on a novel effectiveness measure that scores each approach based on its representational power. Movie-clips of varying complexity are used to underscore the success of the proposed model in real-time.
\end{abstract}

\section{INTRODUCTION}

Although research in building key-frames in video has matured from heuristic selections $[4,5]$ to video content-based approaches $[1,2,3,6]$, the number of keyframes to be selected $\left(N_{k}\right)$ has been either a user-defined constant, or based on heuristic thresholds. However, it is desired to adapt $N_{k}$ with the variation in video-content. This paper proposes an automated, adaptable, online method for computing $N_{k}$, motivated by psychological human visual perception and attention theory.

To emphasize why we need $N_{k}$ to be selected adaptively, suppose a movie is being indexed or key-framed for storyboard type application. One conversation-shot of 5 minutes (9000 frames) with camera mostly focusing on the characters and with minimal motion in the scene, say 10 key-frames may be sufficient. While for a oneminute boxing fight-shot (1800 frames) needs much more than proportionally deduced 2 key-frames to represent the complexity of the visual-information flow. Existing approaches with user-provided "constant-value" $[1,4,5]$ or threshold-based clustering [2] or $\mathrm{P} \%$ criterion in local-minimal motion strategy [3] cannot adapt to this dynamic situations.

Hence a movie-key-framing procedure should take $N_{k}$ computed online, adaptable with the information content of the particular video and motivated by human video-perception psychology. This paper focuses exactly on that. The contributions of this paper are:

(1) An adaptive online method is described to compute "the number of key-frames $\left(N_{k}\right)$ ", motivated by psychological visual perception theory.

(2) The procedure developed in (1) is incorporated in an iterative key-frame selection framework [1] with global (i) color and (ii) motion features (individually) to adapt $N_{k}$.

(3) A novel performance measure is used to compare the proposed method with a clustering based [2] and a motion based [3] approaches.

(4) Experiments are performed with movie-clips of varying complexity for evaluation.

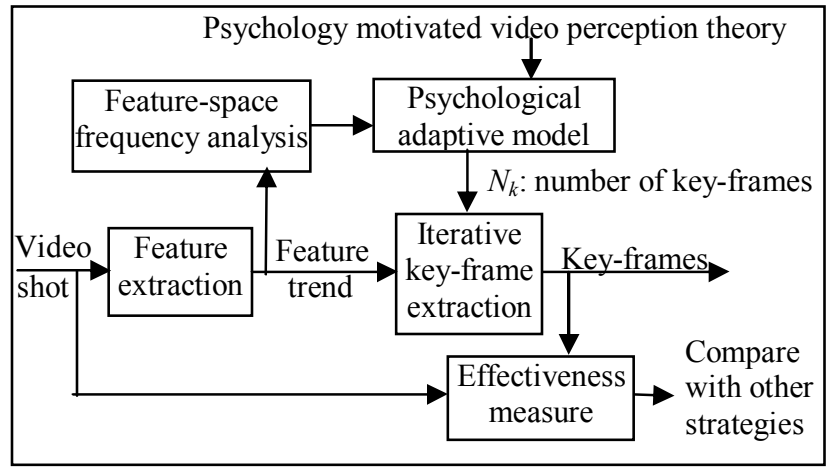

Fig 1: Conceptual diagram of the approach

\section{TECHNICAL APPROACH}

Conceptual diagram of our system is depicted in Fig 1.

\subsection{Automatic selection of number of key-frames, $N_{k}$}

In human visual perception, steady-state visual excitations are less remembered [7]. Larger variation in a movie means richer visual information and hence requires more number of key-frames to meet a minimum representational power (or maximum tolerable error or distortion). We take frame-based features as indicators of visual content of the movie and quantify visual information (content-variation) by taking the Fast Fourier Transform (FFT) of the feature-sequence.

As this work mainly targets movies for human consumption, we utilize general psychological facts regarding human visual perception. According to the "vision and attention" theory in perceptual psychology, when large sequence of video frames (say a standard movie of 1.5-2 Hours) is presented, humans can register 1 frame 
per 30 seconds for slow-paced (with visual content frequency around $0-1 \mathrm{~Hz}$ ) movies [8, 9]. Hence salient movie-shots are generally much larger than 30 seconds and key-frames (for storyboard applications) temporally apart by less than 30 seconds are not practical. The number of frames $K$, a human can at most remember, is (1/30) times the total-time of the movie in seconds $\left(T_{m}\right)$.

$$
\begin{aligned}
& K=(1 / 30) * T_{m} \approx 0.03 * T_{m} \quad \text { where } \\
& T_{m}=\frac{\text { total number of frames }}{\text { frames per second }(\mathrm{fps})}
\end{aligned}
$$

and $f p s=$ frames per second ( 25 for PAL, 30 for NTSC).

Now for faster videos (shots with rapid motion or sports-scenes with video information frequency of more than $2 \mathrm{~Hz}$ ) human memory has to register more number of frames than $K$. Stroud's psychological research established that the time is integrated into perceptual moments of about 100 milliseconds in length [7]; this is the visual signal transmission time from retina to visual-cortex and cognition of the scene using past experience. As a result, although retinal cells have frequency response in the range of $1 \mathrm{MHz}$, general human vision can perceive visual content changes at around $10 \mathrm{~Hz}$. Thus movies are made with 25 (in PAL) or 30 (in NTSC) fps to give illusion of continuity. Even special effects by movie directors are in between $0-30 \mathrm{~Hz}$ and we can safely classify higher frequencies in feature-space as noise. Since steady-state visual excitation $(0-1 \mathrm{~Hz})$ is ignored by human vision [7, $10]$, we consider the range $2-30 \mathrm{~Hz}$ in feature-space and take the strongest frequency $(F)$ as the one representing visual information variation.

We propose that a storyboard of any standard movie formed with properly selected $\left(F^{*} K\right)$ number of keyframes capture almost entire video information. Hence the proposed psychological video-perception model can be written as:

$$
N_{k}=K * F=0.03 * \frac{\text { total number of frames }}{\text { frames per second (fps) }} * F
$$

where $N_{k}$ is number of key-frames to be extracted for $\mathrm{k}^{\text {th }}$ video shot and $F=$ strongest frequency of the featuresequence in 2-30 Hz range. $N_{k}$ is adaptive since $F$ varies with the input.

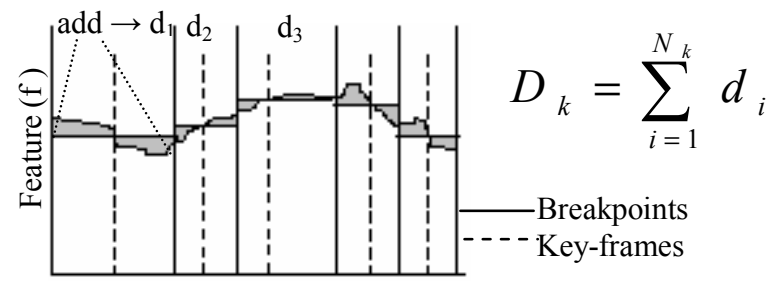

Frame number ( $\mathrm{n}$ )

Fig. 2: Illustration of the frame-feature (f), breakpoints, key-frames and total error $\left(D_{k}\right)$, shown as the shaded area, for a single video-shot.
This online computed $N_{k}$ is plugged into an iterative key-frame extraction approach [1] to make the entire procedure automatic and robust. $N_{k}$ decides the number of key-frames, number of breakpoints $\left(N_{k}+1\right)$, and initialization of breakpoints by equal visual-content criteria. Then these breakpoints and key-frames are optimally [1] placed iteratively to minimize the key-frame-based representational error (Fig. 2).

\subsection{Performance measure}

For evaluation and comparison with other keyframing strategies, we propose a novel performance measure. Key steps are: (1) finding out dissimilarity between a key-frame and the frames it is representing, (2) summing up the dissimilarities for all key-frames over the video-shot and (3) then summing up for all videoshots over the video-clip to define a single index, effectiveness so that key-frames do not under-represent or over-represent the video-clip. The legends used are:

$S=\left\{s_{k} \mid k=1,2, \ldots, M\right\}:$ Collection of shots in the video clip
$s_{k}=\left\{f r_{j} \mid j=\right.$ shot Start $_{k}, \ldots$, shotEnd $\left._{k}\right\} \quad:$ Frames of $\mathbf{k}^{\text {th }}$ video-shot
$\operatorname{keyFr}_{k}=\left\{f r_{n} \mid\left(\right.\right.$ shotStart $_{k} \leq f r_{n} \leq$ shotEnd $\left.\left._{k}\right) \&\left(n=1, \ldots N_{k}\right)\right\}:$ Key-frames

For a key-framing strategy, $N_{k}$ is computed by (2) and key-frames are extracted. Then frame-to-frame dissimilarity is calculated as follows.

$$
\begin{aligned}
& \text { imageDiff }_{n j}=\operatorname{rgb2gray}\left(\text { imgSubtract }\left(\text { fr }_{j}-\text { keyFr }_{n}\right)\right) \\
& \text { diffEnergy }_{n j}=\text { elementWiseSquare (imageDiff }{ }_{n j} \text { ) } \\
& \text { level }=\text { grayThresh }\left(\text { diffEnergy }_{n j}\right) \\
& \text { :adaptive threshold by Otsu's method [11] } \\
& B W=\operatorname{im} 2 b w\left(\text { diffEnergy }_{n j}, \text { level }\right) \quad \text { : binarization }
\end{aligned}
$$

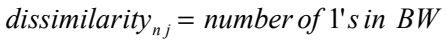

Dissimilarity contributed by an individual key-frame is computed, summed up and normalized to estimate dissimilarity over each video-shot in the movie:

$$
\begin{aligned}
& \text { for } n^{\text {th }} \text { key - frame : } d_{n}=\sum_{j: \text { frames represented by keyF } r_{n}} \text { dissimilar ity }_{n j} \\
& \text { for } k^{\text {th }} \text { video - shot: } D_{k}=\left(\sum_{n=1}^{N_{k}} d_{n}\right) \quad D_{k}^{\prime}=D_{k} / \max _{n}\left(d_{n}\right)
\end{aligned}
$$

Finally representational power of the key-frames over the whole video-clip (collection of $M$ shots) is computed by the effectiveness index as defined below:

$$
\text { Effectiveness }=\left[\sum_{k=1}^{M}\left[1 /\left(1+D_{k}^{\prime}\right)\right]^{L}\right]^{(1 / L)} \text { where, } L=\sum_{k=1}^{M} N_{k}
$$

In equation (4), dissimilarity measure $D_{k}^{\prime}$ decreases and hence similarity measure $\left[\left|1 /\left(1+D_{k}^{\prime}\right)\right| \leq 1\right]$ increases with increasing $N_{k}$. So this performance measure decreases for both very high and very low values of $N_{k}$, and similarly for $L$, the total number of key-frames for the entire movie-clip. Hence, it can be verified that, proposed Effectiveness measure balances between extreme cases of 
under-representation $\left(N_{k} \rightarrow 0\right)$ and over-representation $\left(N_{k} \rightarrow\right.$ total number of frames) of the video-clip.

\section{KEY-FRAMING STRATEGIES COMPARED}

In this paper, we have used color-content variation (Sec 3.1) and inter-frame motion variation ( $\mathrm{Sec} 3.2$ ) as features, and computed fast Fourier transform (FFT) to find $\boldsymbol{F}$ and then $\boldsymbol{N}_{\boldsymbol{k}}$ (as in (2)). $\left(1+N_{k}\right)$ breakpoints $\left(\mathrm{BP}_{i}\right)$ are initialized by equal visual content. Then $N_{k}$ keyframes $\left(k e y F_{i}\right)$ are positioned to minimize errors between corresponding breakpoints. The breakpoints are then updated to minimize distortion between corresponding keyframes. The loop continues until overall distortion remains nearly constant for consecutive iterations (i.e. converged). This guarantees minimum representational error or distortion $\left(D_{k}\right)$, as shown in Fig. 2, (kind of sampling error), by simultaneous optimum (proof in [1]) placement of the breakpoints and key-frames iteratively.

These two strategies have been compared to two other heuristic methods (Sec 3.3.and 3.4).

\subsection{Iterative approach using global color feature}

We have used global average color in the RGB space (for color video, and global intensity average for black-\&white video) as the visual-feature $(f)$ for each frame $(t)$.

$$
f(t)=\frac{\sum_{q: R, G, B} \text { area }\left[\text { hist }\left(t^{\text {th }} \text { frame, } q^{\text {th }} \text { color comp. }\right)\right]}{\text { number of pixels }}
$$

\subsection{Iterative approach using global motion feature}

Motion relates to activity and comes closer to the video-semantics. Global motion (V) is computed from robust phase-based optical flow [12], with horizontal $\left(O_{x}\right)$ and vertical $\left(O_{y}\right)$ motion components, at sampled pixels in $2 \mathrm{D}$ [indexed by $(\mathrm{i}, \mathrm{j})$ ] for $\mathrm{t}^{\text {th }}$ frame [see eq. (6)].

$$
V(t)=\sum_{i} \sum_{j}\left|O_{x}(i, j, t)\right|+\left|O_{y}(i, j, t)\right|
$$

\subsection{Unsupervised clustering}

Intersection of 2D hue-saturation (indices $h$ and $s$ ) histograms $(\mathrm{H})$ is used to compute similarity $(7)$ between two consecutive $\left[\mathrm{r}^{\mathrm{th}}\right.$ and $\left.(\mathrm{r}+1)^{\mathrm{th}}\right]$ frames in HSV space [2]. Incremental clustering uses two heuristic thresholds, cluster-density $(\delta)$ and minimum cluster size $(S z)$. Frames closest to the cluster centers are selected as key-frames.

$$
\operatorname{sim}_{r,(r+1)}=\sum_{h=1}^{16} \sum_{s=1}^{8} \min \left(H_{r}(h, s), H_{r+1}(h, s)\right)
$$

\subsection{Local minima in motion feature trend}

Local minima in global motion [V in (6)] trend of frame sequence emphasize nearby frames [3], and hence they are good candidates for key-frames. To avoid jitters, consecutive minima with more than $P \%$ variation are considered [3]. This $P$ decides $N_{k}$ heuristically.

\section{EXPERIMENTAL RESULTS}

Movie-clips of gradually increasing complexity (in terms of motion-activity, background-changes and camera activity) have been chosen for evaluation of the proposed technique. Data is given in Table 1 with duration in seconds. To emphasize the complexity levels of the different clips, two frames of each clip are shown in Fig. 3. Representational power (as number of key-frames $(\mathbf{L})$, to account under- \& over-representation, and the proposed Effectiveness (E) index) and real-time applicability (in terms of computational time $\left(\mathbf{T}_{\mathbf{C}}\right)$ ) of the extracted key-frames by four techniques in Section 3 are tabulated in Table 2. The threshold values used are:

- Density threshold in clustering: 70000

- $P \%$ criterion in motion based approach: $30 \%$

\begin{tabular}{|c|c|c|}
\hline Clp & Sec & Description \\
\hline 1 & 7 & Volcano eruption: color; very short but fast changes \\
\hline 2 & 16 & $\begin{array}{l}\text { Conversation: } \mathrm{B} \& \mathrm{~W} \text {; slower motions; visible back- } \\
\text { ground changes; camera zooming slowly }\end{array}$ \\
\hline 3 & 21 & Conversation: B\&W; faster motions; one video cut \\
\hline 4 & 30 & $\begin{array}{l}\text { Conversation and movement: color; characters com- } \\
\text { ing in gradually; conversing and moving slowly }\end{array}$ \\
\hline 5 & 27 & $\begin{array}{l}\text { Multi-cut conversation in the dark: color; medium } \\
\text { movements; darkness of the scene }\end{array}$ \\
\hline 6 & 15 & $\begin{array}{l}\text { Fight sequence: color; very fast movements; camera } \\
\text { panning / zooming }\end{array}$ \\
\hline
\end{tabular}

- Shot-detection threshold in color-based iterative approach: 0.25.

- Shot-detection threshold in motion-based iterative approach: 2
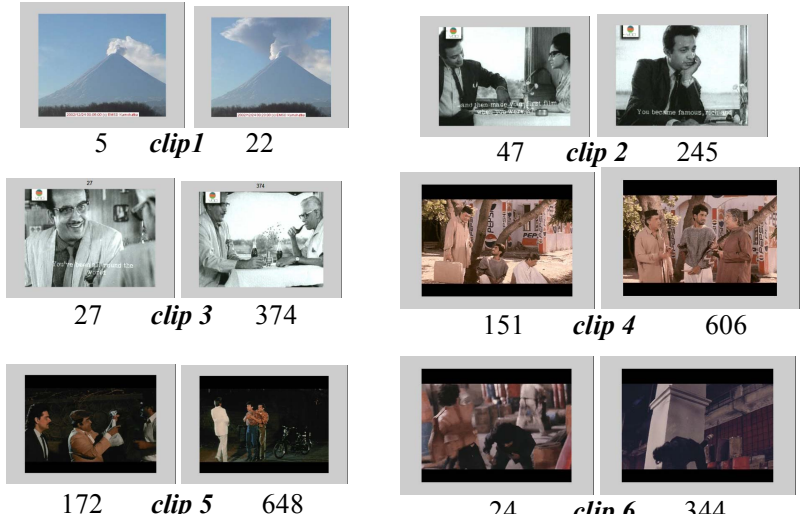

Fig 3: Sample frames of the movie-clips illustrating the widely varying complexity of the data used

Due to space-constraint, only the key-frame results for clip 6 (the most complex one) are shown in Fig. 4. Motion local-minima based technique performs well for movies with slower motion (clip 2, clip 4 \& clip 5) than with faster motion (clip 3 \& clip 6). But considering all the measures $\left(\mathbf{L}, \mathbf{E}, \mathbf{T}_{\mathbf{C}}\right.$ in Table 2), color-feature based iterative approach, using the adaptive online selection of "number of key-frames, $N_{k}$ " proposed in this work, outperforms other techniques, as Effectiveness index (E) is 
high and computational time $\left(\mathbf{T}_{\mathbf{C}}\right)$ is low consistently for all the movie-clips of widely varying complexity.

Table 2: Results

\section{Legends:

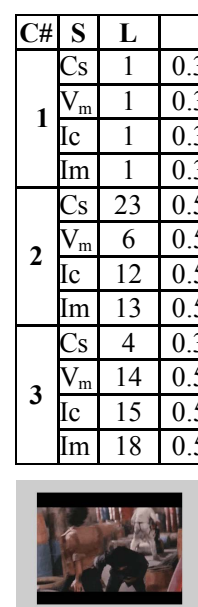

\begin{tabular}{|l|l|l|}
\hline C\# : Clip\# & S: Technique & L: \# of key-frames \\
\hline E: Effectiveness measure & $\mathrm{T}_{\mathrm{C}}$ : Comput. Time (sec) & CS: clustering \\
\hline $\mathrm{V}_{\mathrm{m}}$ : Motion minima & Ic: IterColor & Im: IterMotion \\
\hline
\end{tabular}

26 Ic: IterColor

\begin{tabular}{|c|c|c|c|c|}
\hline C\# & $\mathbf{S}$ & $\mathbf{L}$ & $\mathbf{E}$ & $\mathbf{T}_{\mathrm{C}}$ \\
\hline \multirow{4}{*}{4} & Cs & 2 & 0.1442 & 1512 \\
\hline & $\mathrm{V}_{\mathrm{m}}$ & 9 & 0.5714 & 3783 \\
\hline & Ic & 15 & 0.4887 & 82 \\
\hline & $\operatorname{Im}$ & 20 & 0.4977 & 3803 \\
\hline \multirow{4}{*}{5} & Cs & 11 & 0.4559 & 1372 \\
\hline & $\mathrm{V}_{\mathrm{m}}$ & 27 & 0.5355 & 3482 \\
\hline & Ic & 9 & 0.5206 & 38 \\
\hline & $\mathrm{Im}$ & 20 & 0.4859 & 3485 \\
\hline \multirow{4}{*}{6} & Cs & 4 & 0.3829 & 746 \\
\hline & $\mathrm{V}_{\mathrm{m}}$ & 9 & 0.4903 & 1900 \\
\hline & Ic & 12 & 0.4895 & 15 \\
\hline & $\operatorname{Im}$ & 7 & 0.4151 & 1902 \\
\hline
\end{tabular}

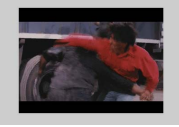

139

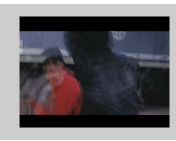

269

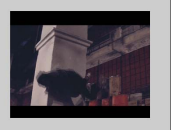

341

4(a): Key-frames by Clustering

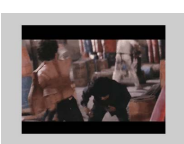

24

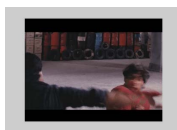

204

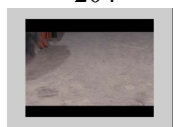

356

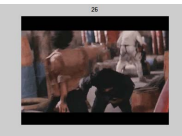

26

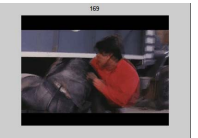

169

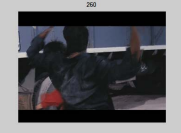

260

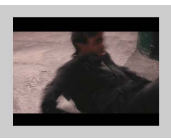

72

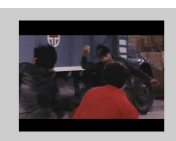

104

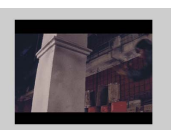

302

4(b): Key-frames by motion local minima technique

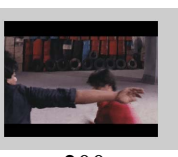

200

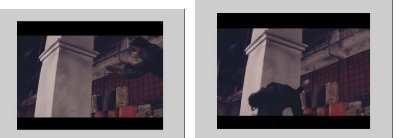

344

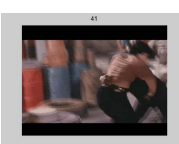

41

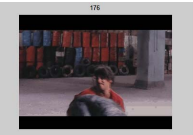

176

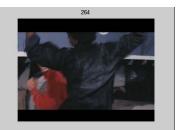

264

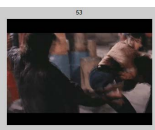

53

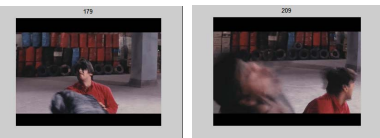

179

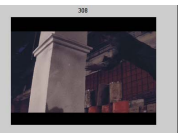

308

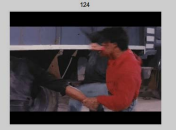

124
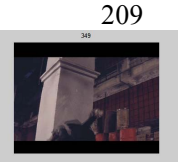

349 4(c): Key-frame by global color feature \& iterative color algorithm.

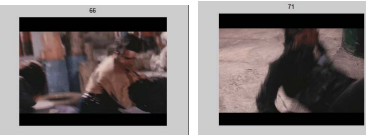

66

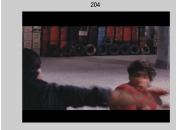

204

71

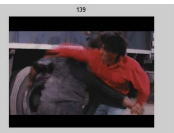

139

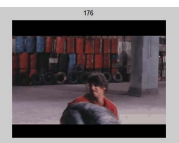

176

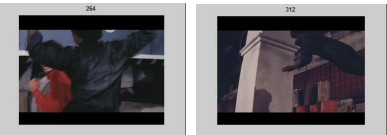

312

4(d): Key-frames by global motion feature \& iterative motion algorithm.

Fig. 4: Results for Clip 6 (fight-sequence)

\section{CONCLUSIONS}

We have proposed a novel psychological model to compute the number of key-frames $\left(N_{k}\right)$ to be extracted in a dynamic and adaptive way according to the information content of the movie clip. The approach is validated with four key-frame selection approaches, two adaptive and iterative strategies based on global color and global motion features and two heuristic strategies based on colorhistogram based clustering and local-minima in motion trend.

We emphasize here that the present work has been targeted for commercial movie-clips developing keyframes for forming storyboard-type briefing. Application for laboratory video-data, like mere camera panning or earthquake sequences requires structural features. Such sophistications using learning based techniques are topics of future research.

\section{REFERENCES}

[1] H.-C. Lee \& S.-D. Kim, "Iterative key-frame selection in the rate-constraint environment," Sig. Proc.: Im. Comm.: 18 (2003), 1-15.

[2] Y. Zhuang, Y. Rui, S.-T. Huang and S. Mehrotra, "Adaptive key frame extraction using unsupervised clustering," Proc. ICIP: 1 (1998) pp 866-870.

[3] W. Wolf, "Key frame selection by motion analysis," ICASSP: (1996) pp 1228-1231.

[4] M.M. Yeung and B. Liu, "Efficient matching and clustering of video shots," in Proc. ICIP: (1995) pp 338-342.

[5] R. Zabih, J. Miller and K. Mai, "A feature-based algorithm for detecting and classifying scene breaks," in Proc. ACM Multimedia: (1995) pp 189-200.

[6] Z. Rasheed and M. Shah, "Scene detection in Hollywood movies and TV shows," in Proc. CVPR: (2003), Vol. 2, pp 343-348.

[7] R.N. Haber and M. Hershenson, "The Psychology of Visual Perception," Holt, Rinehart and Winston Inc.: (1973).

[8] J.M. Findlay and I.D. Gilchrist, "Active Vision: the Psychology of Looking and Seeing," Oxford University Press: (2003).

[9] R.D. Wright (Ed.), "Visual Attention", Oxf. Univ. Press: (1998).

[10] S. Soraci Jr. and K.M. Soraci (Ed.), "Visual Information Processing," Praeger Pub.: (2003).

[11] N. Otsu, "A threshold selection method from gray-level histogram," IEEE Trans. on Sys. Man and Cyb. : (1979) Vol. 9, No. 1, pp 62-66.

[12] T. Gautama and M.M. Van Hulle, "A phase-based approach to the estimation of the optical flow field using spatial filtering," in IEEE Trans. on Neural Nets: (2002) Vol. 13(5), pp 1127-1136. 